Moroccan J. of Pure and Appl. Anal. (MJPAA)

Volume 4(1), 2018, Pages 9-16

ISSN: Online 2351-8227 - Print 2605-6364

DOI 10.1515/mjpaa-2018-0002

\title{
On Soft Real Point Matrices and Their Operations
}

\author{
S. Hussain ${ }^{1, a}$, H. F. AkIz ${ }^{2}$ And A. I. Alajlan ${ }^{1}$
}

ABSTRACT. In this paper, we define and investigate soft real point matrices and their operations which are more functional to make theoretical studies in the soft real point set theory. We then define products of soft real point matrices and their properties. Examples are also provided to validate the existence of defined notions.

Key words and phrases. Soft single points, Soft real points, Soft real point matrix.

\section{Introduction}

Zadeh [10] introduced the notion of fuzzy sets. Many researchers worked on the generalization of fuzzy sets. One of the generalization of fuzzy sets, Molodtsov [8] initiated the concept of soft set theory as a new mathematical tool for modelling vagueness and uncertainties inherent in the problems of engineering, physical science, biological science, economics, social science, medical science, etc. For more details on studies as well as structures of soft sets and fuzzy soft sets, the interested reader is refer to [1-5],[7-11].

During the study of soft topological spaces and in particular the soft version of soft usual topology of real numbers, the notion of soft single points and soft real points are introduced in [6]. In [6], Hussain et.al also defined the basic operations on soft real points and explored the algebraic properties. It is observed that the set of all soft real points forms a ring. It is worth mentioning that these soft real points may extend to several important fixed point theorems deduce from comparable existing results.

Now we define and investigate soft real point matrices and their operations which are more functional to make theoretical studies in the soft real point set theory. We then define products of soft real point matrices and their properties in details.

\section{Preliminaries}

To make our paper self contained, we recall some definitions.

Received October 10, 2018 - Accepted December 4, 2018.

(C) The Author(s) 2017. This article is published with open access by Sidi Mohamed Ben Abdallah University.

${ }^{1}$ Department of Mathematics, College of Science, Qassim University, P.O. Box 6644, Buraydah 51482, Saudi Arabia.

${ }^{2}$ Department of Mathematics, Bozok University Yozgat 66900, Turkey.

${ }^{a}$ e-mail: sabiriub@yahoo.com; sh.hussain@qu.edu.sa . 
Definition 2.1. ([8]) A pair $(F, A)$ is called a soft set over $X$, where $F$ is a mapping given by $F: A \rightarrow P(X)$. In other words, a soft set over $X$ is a parameterized family of subsets of the universe $X$.

Definition 2.2. ([3]) Let $(F, A)$ be a soft set over $X$. If $F(x)=\emptyset$, for all $x \in A$, then $(F, A)$ is called an empty soft set, denoted by $\Phi_{A}$.

Definition 2.3. ([3]) Let $(F, A)$ be a soft set over $X$. If $F(x)=X$, for all $x \in A$, then $(F, A)$ is called an A-universal(Absolute) soft set, denoted by $F_{\widetilde{A}}$.

Definition 2.4. ([7]) Let $(F, A)$ and $(G, B)$ are soft sets over $X$. Then $(F, A)$ is a soft subset of $(G, B)$, denoted by $(F, A) \widetilde{\subseteq}(G, B)$, if $F(x) \subseteq G(x)$, for all $x \in A$.

Similarly $(F, A)$ is called a soft superset of $(G, B)$, if $(G, B)$ is a soft subset of $(F, A)$. This relation is denoted by $(F, A) \supseteq(G, B)$.

Definition 2.5. ([7]) Two soft sets $(F, A)$ and $(G, B)$ over a common universe $X$ are called soft equal if $(F, A) \widetilde{\subseteq}(G, B)$ and $(G, B) \widetilde{\subseteq}(F, A)$.

Definition 2.6. ([7]) The union of two soft sets of $(F, A)$ and $(G, B)$ over the common universe $U$ is the soft set $(H, C)$, where $C=A \cup B$ and for all $x \in C$,

$$
H(x)=\left\{\begin{array}{cl}
F(x), & \text { if } x \in A-B \\
G(x), & \text { if } x \in B-A \\
F(x) \cup G(x), & \text { if } x \in A \cap B
\end{array}\right.
$$

We write $(F, A) \cup(G, B)=(H, C)$.

Definition 2.7. ([7]) The intersection $(H, C)$ of two soft sets $(F, A)$ and $(G, B)$ over a common universe $U$, denoted $(F, A) \cap(G, B)$, is defined as $C=A \cap B$, and $H(x)=F(x) \cap G(x)$, for all $x \in C$.

Definition 2.8. ([6]) Let $X \neq \Phi$ be a set. Then for each $x \in X$ and $A \subseteq X$, we define functions $F_{x}^{A}:\{x\} \rightarrow P(X),(x \mapsto A$. $)$

Each function $F_{x}^{A}$ is called a fixed point function of the pair $x$ and $A$. The set $\left\{\left(x, F_{x}^{A}(x)\right)\right\}=\{(x, A)\}$ is called a soft single point set on $X$.

Also, for all $x \in X$, the set $\{(x, \Phi)\}$ is called empty soft single point set.

The set of all soft single points is denoted by

$$
\mathcal{S}_{X}=\left\{\left\{\left(x, F_{x}^{A}(x)\right)\right\} \mid x \in X, A \subseteq X\right\} .
$$

Notice that each function $F_{x}^{A}$ is unique and different for each $x \in X, \quad A \subseteq X$ and consists of one element $(x, A)$.

Proposition 2.1. ([6]) If $X \neq \Phi$ is a set, then the number of soft single points is determined by: $\left|\mathcal{S}_{X}\right|=n 2^{n}$, where $n$ is the number of elements of $X$.

Example 2.1. Let $X=\{1,2,3\}$ be a set. Then for each $x \in X$, soft single point sets are following:

$$
\begin{aligned}
& \{(1, X)\},\{(2, X)\},\{(3, X)\},\{(1,\{1\})\},\{(1,\{1,2\})\},\{1,\{1,3\}\}, \\
& \{(2,\{2\})\},\{(2,\{2,3\})\},\{(3,\{2,3\})\},\{(3,\{1,3\})\},\{(2,\{1,2\})\} \\
& \{(2,\{1\})\},\{(2,\{3\})\},\{(1,\{2\})\},\{(1,\{3\})\},\{(2,\{1,3\})\},\{(3,\{1,2\})\}, \\
& \{(3,\{1\})\},\{(3,\{3\})\},\{(3,\{2\})\},\{(1,\{2,3\}\}, \\
& \{(1, \emptyset)\},\{(2, \emptyset)\},\{(3, \emptyset)\} .
\end{aligned}
$$

Definition 2.9. ([6]) If $x=x^{\prime}$ and $A=A^{\prime}$, for $x, x^{\prime} \in X$ and $A, A^{\prime} \subseteq X$, then we say that $\{(x, A)\}$ and $\left\{\left(x^{\prime}, A^{\prime}\right)\right\}$ are equal and denoted by $\{(x, A)\}=\left\{\left(x^{\prime}, A^{\prime}\right)\right\}$.

Definition 2.10. ([6]) Let $\mathcal{R}$ be the set of real numbers. Then we get soft single point sets by using fixed point functions in Definition 2.8. For all $r \in \mathcal{R}$ and $A \subseteq \mathcal{R}, F_{r}^{A}:\{r\} \rightarrow P(\mathcal{R}), r \mapsto A$.

Then the set $\widetilde{\mathcal{R}}=\{\{(r, A)\} \mid r \in \mathcal{R}, A \subseteq \mathcal{R}\}$ is called the set of soft real points.

Example 2.2. $\{(1,(0,1))\},\left\{\left(\frac{1}{2},(5, \infty)\right)\right\},\{(\sqrt{3},\{0\})\}$ are the examples of soft real points. 
Definition 2.11. ([6]) If $r=r^{\prime}$ and $A=A^{\prime}$, for $r, r^{\prime} \in \mathcal{R}$ and $A, A^{\prime} \subseteq \mathcal{R}$, then we say that soft real points $\{(r, A)\}$ and $\left\{\left(r^{\prime}, A^{\prime}\right)\right\}$ are equal, if $\{(r, A)\}=\left\{\left(r^{\prime}, A^{\prime}\right)\right\}$.

\section{Soft Real Point Matrices and their Operations}

In this section, we define and investigate soft real point matrices and their operations which are more functional to make theoretical studies in the soft real point set theory. We then define products of soft real point matrices and their properties.

Definition 3.1. Let $\mathcal{R}$ be the set of real numbers, $A \subseteq \mathcal{R}$ and $P(A)$ be the power set of $A$. Then soft real points on $A$ are defined by $F_{r}^{A}:\{r\} \rightarrow P(A), r \in A$.

Then $\widetilde{\mathcal{R}}_{A}=\{\{(r, u)\}$, for some $r \in A$ and $u \subseteq A\}$ be the soft subset real points of $\widetilde{\mathcal{R}}$. Also, we can define the characters function of $\widetilde{\mathcal{R}}_{A}$ by

$$
X_{\widetilde{\mathcal{R}}_{A}}: \widetilde{\mathcal{R}}_{A} \rightarrow\{0,1\}, X_{\widetilde{\mathcal{R}}_{A}}\{(r, u)\}= \begin{cases}1, & \text { if }\{(r, u)\} \in \widetilde{\mathcal{R}}_{A} \\ 0, & \text { if }\{(r, u)\} \notin \widetilde{\mathcal{R}}_{A}\end{cases}
$$

Now we define the Matrix on $\widetilde{\mathcal{R}}_{A}$ denoted by $\left[\widetilde{\mathcal{R}}_{A}\right]$ as follows:

$$
\begin{aligned}
& \begin{array}{lll}
u_{1} & u_{2} & u_{n}
\end{array} \\
& {\left[\widetilde{\mathcal{R}}_{A}\right]=\begin{array}{c}
r_{1} \\
r_{2} \\
: \\
r_{m}
\end{array}\left[\begin{array}{ccccc}
X_{\widetilde{\mathcal{R}}_{A}}\left\{\left(r_{1}, u_{1}\right)\right\} & X_{\widetilde{\mathcal{R}}_{A}}\left\{\left(r_{1}, u_{2}\right)\right\} & & X_{\widetilde{\mathcal{R}}_{A}}\left\{\left(r_{1}, u_{n}\right)\right\} \\
X_{\widetilde{\mathcal{R}}_{A}}\left\{\left(r_{2}, u_{1}\right)\right\} & \ldots & . . & : \\
X_{\widetilde{\mathcal{R}}_{A}}\left\{\left(r_{m}, u_{1}\right)\right\} & . . & . . & : \\
& . . & . . & X_{\widetilde{\mathcal{R}}_{A}}\left\{\left(r_{m}, u_{n}\right)\right\}
\end{array}\right]}
\end{aligned}
$$

We arrange the rows on the real numbers of $A$ from the smaller to larger and arrange the columns on all subsets of A. (i.e. from $P(A))$.

If $a_{i j}=X_{\widetilde{\mathcal{R}}_{A}}\left\{\left(r_{i}, u_{j}\right)\right\}$ we write the matrix $\left[\widetilde{\mathcal{R}}_{A}\right]$ as

$$
\left[\widetilde{\mathcal{R}}_{A}\right]=\left[\begin{array}{cccc}
a_{11} & a_{12} & . . & a_{1 n} \\
a_{21} & : & : & : \\
: & : & : & : \\
a_{m 1} & . . & . . & a_{m n}
\end{array}\right]
$$

and is called $m \times n$ soft real point matrix on $\widetilde{\mathcal{R}}_{A}$. The set of all $m \times n$ soft real point matrices over $\widetilde{\mathcal{R}}$ will be denoted by $M(\widetilde{\mathcal{R}})_{m \times n}$.

Example 3.1. Let $A=\{1,2\} \subset \mathcal{R}$ and $P(A)=\{\Phi, A,\{1\},\{2\}\}$. If we consider a set of soft real point on $A$ as:

$\left.\widetilde{\mathcal{R}}_{A}=\{\{(1, \Phi)\},\{(1,\{1\})\},\{(1,\{2\})\},\{(2,\{1\})\},\{(2,\{2\})\},(2, A)\}\right\}$. Then the corresponding soft real point matrix $\left[\widetilde{\mathcal{R}}_{A}\right]$ is written as:

$$
\begin{aligned}
& {\left[\widetilde{\mathcal{R}}_{A}\right]=\left[\begin{array}{cccc}
X_{\widetilde{\mathcal{R}}_{A}}\{(1, \Phi)\} & X_{\widetilde{\mathcal{R}}_{A}}\{(1,\{1\})\} & X_{\widetilde{\mathcal{R}}_{A}}\{(1,\{2\})\} & X_{\widetilde{\mathcal{R}}_{A}}\{(1, A)\} \\
X_{\widetilde{\mathcal{R}}_{A}}\{(2, \Phi)\} & X_{\widetilde{\mathcal{R}}_{A}}\{(2,\{1\})\} & X_{\widetilde{\mathcal{R}}_{A}}\{(1,\{2\})\} & X_{\widetilde{\mathcal{R}}_{A}}\{(2, A)\}
\end{array}\right]} \\
& =\left[\begin{array}{cccc}
1 & 1 & 1 & 0 \\
0 & 1 & 1 & 1
\end{array}\right]
\end{aligned}
$$

Proposition 3.1. Let $\left[\widetilde{\mathcal{R}}_{A}\right] \in M(\widetilde{\mathcal{R}})_{m \times n}$. Then $\left[\widetilde{\mathcal{R}}_{A}\right]$ is called

(1) A zero soft real point matrix denoted by $[\widetilde{0}]$, if $a_{i j}=0$, for all $i$ and $j$.

(2) An A-universal(Absolute) soft real point matrix denoted by $[\widetilde{1}]$, if $a_{i j}=1$, for all $i$ and $j$. 
Proposition 3.2. Let $\left[\widetilde{\mathcal{R}}_{A}\right],\left[\widetilde{\mathcal{R}}_{B}\right] \in M(\widetilde{\mathcal{R}})_{m \times n}$. Then

(1) $\left[\widetilde{\mathcal{R}}_{A}\right]$ is a soft real point submatix of $\left[\widetilde{\mathcal{R}}_{B}\right]$, denoted by $\left[\widetilde{\mathcal{R}}_{A}\right] \widetilde{\subseteq}\left[\widetilde{\mathcal{R}}_{B}\right]$, if $a_{i j} \leq b_{i j}$, for all $i$ and $j$.

(2) $\left[\widetilde{\mathcal{R}}_{A}\right]$ and $\left[\widetilde{\mathcal{R}}_{B}\right]$ are soft real point equal matrices, denoted by $\left[\widetilde{\mathcal{R}}_{A}\right] \cong\left[\widetilde{\mathcal{R}}_{B}\right]$, if $a_{i j}=b_{i j}$, for all $i$ and $j$.

(3) The common sum of $\left[\widetilde{\mathcal{R}}_{A}\right]$ and $\left[\widetilde{\mathcal{R}}_{B}\right]$ is $\left[\widetilde{\mathcal{R}}_{A}\right] \widetilde{+}\left[\widetilde{\mathcal{R}}_{B}\right] \cong\left[\widetilde{\mathcal{R}}_{C}\right]$, where $c_{i j}=\max \left\{a_{i j}, b_{i j}\right\}$, for all $i$ and $j$.

(4) The common different of $\left[\widetilde{\mathcal{R}}_{A}\right]$ and $\left[\widetilde{\mathcal{R}}_{B}\right]$ is $\left[\widetilde{\mathcal{R}}_{A}\right] \simeq\left[\widetilde{\mathcal{R}}_{B}\right] \cong\left[\widetilde{\mathcal{R}}_{C}\right]$, where $c_{i j}=\min \left\{a_{i j}, b_{i j}\right\}$, for all $i$ and $j$.

(5) Complement of $\left[\widetilde{\mathcal{R}}_{A}\right]$, denoted by $\left[\widetilde{\mathcal{R}}_{A}\right]^{c} \cong\left[\widetilde{\mathcal{R}}_{C}\right]$, where $c_{i j}=1-a_{i j}$, for all $i$ and $j$.

Example 3.2. Let $\left[\widetilde{\mathcal{R}}_{A}\right]$ be the soft real point matrix as in Example 3.1 and let $B=\{2,3\}$, then $P(B)=$ $\{\Phi, B,\{2\},\{3\}\}$. If we consider a set of soft real point on $B$ as :

$\widetilde{\mathcal{R}}_{B}=\{\{(2, \Phi)\},\{(2, B)\},\{(3,\{2\})\},\{(3,\{3\})\},\{(3, B)\}\}$, then the soft real point matrix $\left[\widetilde{\mathcal{R}}_{B}\right]$ is written by $\left[\widetilde{\mathcal{R}}_{B}\right]=\left[\begin{array}{llll}1 & 0 & 0 & 1 \\ 0 & 1 & 1 & 1\end{array}\right]$. Then

$\left[\widetilde{\mathcal{R}}_{A}\right] \widetilde{+}\left[\widetilde{\mathcal{R}}_{B}\right]=\left[\begin{array}{llll}1 & 1 & 1 & 1 \\ 0 & 1 & 1 & 1\end{array}\right],\left[\widetilde{\mathcal{R}}_{A}\right] \simeq\left[\widetilde{\mathcal{R}}_{B}\right]=\left[\begin{array}{llll}1 & 0 & 0 & 0 \\ 0 & 1 & 1 & 1\end{array}\right]$ and

$\left[\widetilde{\mathcal{R}}_{A}\right]^{c}=\left[\begin{array}{llll}0 & 0 & 0 & 1 \\ 1 & 0 & 0 & 0\end{array}\right]$

Proposition 3.3. Let $\left[\widetilde{\mathcal{R}}_{A}\right] \in M(\widetilde{\mathcal{R}})_{m \times n}$. Then

(i) $\left[\left[\widetilde{\mathcal{R}}_{A}\right]^{c}\right]^{c}=\left[\widetilde{\mathcal{R}}_{A}\right]$.

(ii) $[\widetilde{0}]^{c}=[\widetilde{1}]$.

(iii) $[\widetilde{1}]^{c}=[\widetilde{0}]$

Proof. (i) Let $\left[\widetilde{\mathcal{R}}_{A}\right]^{c}=\left[\widetilde{\mathcal{R}}_{C}\right]$, where $c_{i j}=1-a_{i j}$, for all $i, j$.

Then $\left[\left[\widetilde{\mathcal{R}}_{A}\right]^{c}\right]^{c}=\left[\widetilde{\mathcal{R}}_{C}\right]^{c}$, where $\left(c_{i j}\right)^{c}=\left(1-a_{i j}\right)^{c}$, for all $i, j$.

So, $\left(c_{i j}\right)^{c}=\left(1-a_{i j}\right)^{c}=1-\left(1-a_{i j}\right)=a_{i j}$, for all $i, j$.

Hence, $\left[\left[\widetilde{\mathcal{R}}_{A}\right]^{c}\right]^{c}=a_{i j}=\left[\widetilde{\mathcal{R}}_{A}\right]$.

(ii) Let $[\widetilde{0}]^{c}=\left[c_{i j}\right]$, where $c_{i j}=1-0$, for all $i, j$. Then $c_{i j}=1$, for all $i, j$.

So, $\left[c_{i j}\right]=[\widetilde{1}]$. Hence $[\widetilde{0}]^{c}=[\widetilde{1}]$.

(iii) Similar to $($ ii $)$

The proofs of the following propositions $3.4,3.5,3.6$ and 3.7 directly follow from the definition and are thus omitted.

Proposition 3.4. Let $\left[\widetilde{\mathcal{R}}_{A}\right],\left[\widetilde{\mathcal{R}}_{B}\right],\left[\widetilde{\mathcal{R}}_{D}\right] \in M(\widetilde{\mathcal{R}})_{m \times n}$. Then

(i) $\left[\widetilde{\mathcal{R}}_{A}\right] \widetilde{\subseteq}[\widetilde{1}]$.

(ii) $[\widetilde{0}] \widetilde{\subseteq}\left[\widetilde{\mathcal{R}}_{A}\right]$.

(iii) $\left[\widetilde{\mathcal{R}}_{A}\right] \widetilde{\simeq}\left[\widetilde{\mathcal{R}}_{A}\right]$.

(iv) $\left[\widetilde{\mathcal{R}}_{A}\right] \widetilde{\subseteq}\left[\widetilde{\mathcal{R}}_{B}\right]$ and $\left[\widetilde{\mathcal{R}}_{B}\right] \widetilde{\subseteq}\left[\widetilde{\mathcal{R}}_{D}\right] \Rightarrow\left[\widetilde{\mathcal{R}}_{A}\right] \widetilde{\subset}\left[\widetilde{\mathcal{R}}_{D}\right]$.

Proposition 3.5. Let $\left[\widetilde{\mathcal{R}}_{A}\right],\left[\widetilde{\mathcal{R}}_{B}\right],\left[\widetilde{\mathcal{R}}_{D}\right] \in M(\widetilde{\mathcal{R}})_{m \times n}$. Then 
(i) $\left[\widetilde{\mathcal{R}}_{A}\right] \cong\left[\widetilde{\mathcal{R}}_{B}\right]$ and $\left[\widetilde{\mathcal{R}}_{B}\right] \cong\left[\widetilde{\mathcal{R}}_{D}\right] \Longleftrightarrow\left[\widetilde{\mathcal{R}}_{A}\right] \cong\left[\widetilde{\mathcal{R}}_{D}\right]$.

(ii) $\left[\widetilde{\mathcal{R}}_{A}\right] \widetilde{\simeq}\left[\widetilde{\mathcal{R}}_{B}\right]$ and $\left[\widetilde{\mathcal{R}}_{B}\right] \widetilde{\simeq}\left[\widetilde{\mathcal{R}}_{A}\right] \Longleftrightarrow\left[\widetilde{\mathcal{R}}_{A}\right] \cong\left[\widetilde{\mathcal{R}}_{B}\right]$.

Proposition 3.6. Let $\left[\widetilde{\mathcal{R}}_{A}\right],\left[\widetilde{\mathcal{R}}_{B}\right],\left[\widetilde{\mathcal{R}}_{D}\right] \in M(\widetilde{\mathcal{R}})_{m \times n}$. Then

(i) $\left[\widetilde{\mathcal{R}}_{A}\right] \widetilde{+}\left[\widetilde{\mathcal{R}}_{A}\right] \cong\left[\widetilde{\mathcal{R}}_{A}\right]$.

(ii) $\left[\widetilde{\mathcal{R}}_{A}\right] \tilde{+}[\widetilde{0}] \cong\left[\widetilde{\mathcal{R}}_{A}\right]$.

(iii) $\left[\widetilde{\mathcal{R}}_{A}\right] \widetilde{+}[\widetilde{1}] \cong[\widetilde{1}]$.

(iv) $\left[\widetilde{\mathcal{R}}_{A}\right] \widetilde{+}\left[\widetilde{\mathcal{R}}_{A}\right]^{c}=[\widetilde{1}]$.

(v) $\left[\widetilde{\mathcal{R}}_{A}\right] \tilde{+}\left[\widetilde{\mathcal{R}}_{B}\right]=\left[\widetilde{\mathcal{R}}_{B}\right] \tilde{+}\left[\widetilde{\mathcal{R}}_{A}\right]$.

(vi) $\left(\left[\widetilde{\mathcal{R}}_{A}\right] \widetilde{+}\left[\widetilde{\mathcal{R}}_{B}\right]\right) \widetilde{+}\left[\widetilde{\mathcal{R}}_{D}\right]=\left[\widetilde{\mathcal{R}}_{A}\right] \widetilde{+}\left(\left[\widetilde{\mathcal{R}}_{B}\right] \widetilde{+}\left[\widetilde{\mathcal{R}}_{D}\right]\right)$.

Proposition 3.7. Let $\left[\widetilde{\mathcal{R}}_{A}\right],\left[\widetilde{\mathcal{R}}_{B}\right],\left[\widetilde{\mathcal{R}}_{D}\right] \in M(\widetilde{\mathcal{R}})_{m \times n}$. Then

(i) $\left[\widetilde{\mathcal{R}}_{A}\right] \simeq\left[\widetilde{\mathcal{R}}_{A}\right] \cong\left[\widetilde{\mathcal{R}}_{A}\right]$.

(ii) $\left[\widetilde{\mathcal{R}}_{A}\right] \simeq[\widetilde{0}] \cong[\widetilde{0}]$.

(iii) $\left[\widetilde{\mathcal{R}}_{A}\right] \simeq[\widetilde{1}] \cong\left[\widetilde{\mathcal{R}}_{A}\right]$.

(iv) $\left[\widetilde{\mathcal{R}}_{A}\right] \simeq\left[\widetilde{\mathcal{R}}_{A}\right]^{c}=[\widetilde{0}]$.

(v) $\left[\widetilde{\mathcal{R}}_{A}\right] \simeq\left[\widetilde{\mathcal{R}}_{B}\right]=\left[\widetilde{\mathcal{R}}_{B}\right] \simeq\left[\widetilde{\mathcal{R}}_{A}\right]$

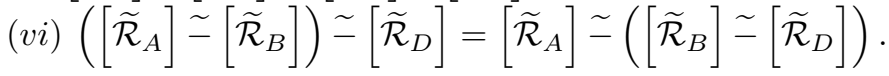

Now we prove the Demorgan's law for soft real point matrices.

Proposition 3.8. Let $\left[\widetilde{\mathcal{R}}_{A}\right],\left[\widetilde{\mathcal{R}}_{B}\right] \in M(\widetilde{\mathcal{R}})_{m \times n}$. Then

(i) $\left[\left[\widetilde{\mathcal{R}}_{A}\right] \widetilde{+}\left[\widetilde{\mathcal{R}}_{B}\right]\right]^{c}=\left[\widetilde{\mathcal{R}}_{A}\right]^{c} \simeq\left[\widetilde{\mathcal{R}}_{B}\right]^{c}$.

(ii) $\left[\left[\widetilde{\mathcal{R}}_{A}\right] \simeq\left[\widetilde{\mathcal{R}}_{B}\right]\right]^{c}=\left[\widetilde{\mathcal{R}}_{B}\right]^{c} \widetilde{+}\left[\widetilde{\mathcal{R}}_{A}\right]^{c}$.

Proof. (i) For all $i$ and $j$,

$\left[\left[\widetilde{\mathcal{R}}_{A}\right] \widetilde{+}\left[\widetilde{\mathcal{R}}_{B}\right]\right]^{c}=\left[\max \left\{a_{i j}, b_{i j}\right\}\right]^{c}=\left[1-\max \left\{a_{i j}, b_{i j}\right\}\right]$

$=\left[\min \left\{1-a_{i j}, 1-b_{i j}\right\}\right]=\left[\widetilde{\mathcal{R}}_{A}\right]^{c} \simeq\left[\widetilde{\mathcal{R}}_{B}\right]^{c}$.

(ii) Similarly to $(i)$

The following example justify the above proposition.

Example 3.3. We consider the example as presented in Example 4.

Then $\left[\left[\widetilde{\mathcal{R}}_{A}\right] \widetilde{+}\left[\widetilde{\mathcal{R}}_{B}\right]\right]^{c}=\left[\begin{array}{llll}0 & 0 & 0 & 0 \\ 1 & 0 & 0 & 0\end{array}\right]$ and $\left[\widetilde{\mathcal{R}}_{B}\right]^{c}=\left[\begin{array}{llll}0 & 1 & 1 & 0 \\ 1 & 0 & 0 & 0\end{array}\right]$.

So,

$\left[\left[\widetilde{\mathcal{R}}_{A}\right] \widetilde{+}\left[\widetilde{\mathcal{R}}_{B}\right]\right]^{c}=\left[\begin{array}{llll}0 & 0 & 0 & 0 \\ 1 & 0 & 0 & 0\end{array}\right]=\left[\widetilde{\mathcal{R}}_{A}\right]^{c} \simeq\left[\widetilde{\mathcal{R}}_{B}\right]^{c}=\left[\begin{array}{llll}0 & 0 & 0 & 0 \\ 1 & 0 & 0 & 0\end{array}\right]$,

$\left[\left[\widetilde{\mathcal{R}}_{A}\right] \simeq\left[\widetilde{\mathcal{R}}_{B}\right]\right]^{c}=\left[\begin{array}{llll}0 & 1 & 1 & 1 \\ 1 & 0 & 0 & 0\end{array}\right]=\left[\widetilde{\mathcal{R}}_{B}\right]^{c} \widetilde{+}\left[\widetilde{\mathcal{R}}_{A}\right]^{c}=\left[\begin{array}{llll}0 & 1 & 1 & 1 \\ 1 & 0 & 0 & 0\end{array}\right]$ 
The following proposition gives us the associative property of soft real point matrices in terms of common sum and common difference operation.

Proposition 3.9. Let $\left[\widetilde{\mathcal{R}}_{A}\right],\left[\widetilde{\mathcal{R}}_{B}\right],\left[\widetilde{\mathcal{R}}_{D}\right] \in M(\widetilde{\mathcal{R}})_{m \times n}$. Then

(i) $\left[\widetilde{\mathcal{R}}_{A}\right] \widetilde{+}\left(\left[\widetilde{\mathcal{R}}_{B}\right] \simeq\left[\widetilde{\mathcal{R}}_{D}\right]\right)=\left(\left[\widetilde{\mathcal{R}}_{A}\right] \widetilde{+}\left[\widetilde{\mathcal{R}}_{B}\right]\right) \simeq\left(\left[\widetilde{\mathcal{R}}_{A}\right] \widetilde{+}\left[\widetilde{\mathcal{R}}_{D}\right]\right)$.

(ii) $\left[\widetilde{\mathcal{R}}_{A}\right] \simeq\left(\left[\widetilde{\mathcal{R}}_{B}\right] \widetilde{+}\left[\widetilde{\mathcal{R}}_{D}\right]\right)=\left(\left[\widetilde{\mathcal{R}}_{A}\right] \stackrel{\sim}{-}\left[\widetilde{\mathcal{R}}_{B}\right]\right) \widetilde{+}\left(\left[\widetilde{\mathcal{R}}_{A}\right] \simeq\left[\widetilde{\mathcal{R}}_{D}\right]\right)$.

The following example validate the above proposition.

Example 3.4. Consider the following three soft real point matrices $\left[\widetilde{\mathcal{R}}_{A}\right],\left[\widetilde{\mathcal{R}}_{B}\right]$ and $\left[\widetilde{\mathcal{R}}_{D}\right]$ as:

$$
\begin{aligned}
& {\left[\widetilde{\mathcal{R}}_{A}\right]=\left[\begin{array}{llll}
1 & 0 & 0 & 0 \\
0 & 1 & 1 & 0
\end{array}\right],\left[\widetilde{\mathcal{R}}_{B}\right]=\left[\begin{array}{llll}
1 & 1 & 1 & 1 \\
0 & 1 & 0 & 1
\end{array}\right] \text { and }} \\
& {\left[\widetilde{\mathcal{R}}_{D}\right]=\left[\begin{array}{llll}
0 & 0 & 0 & 1 \\
1 & 0 & 1 & 0
\end{array}\right] \text {. Then }} \\
& {\left[\left[\widetilde{\mathcal{R}}_{B}\right] \simeq\left[\widetilde{\mathcal{R}}_{D}\right]\right]=\left[\begin{array}{llll}
0 & 0 & 0 & 1 \\
0 & 0 & 0 & 0
\end{array}\right] \text { and }} \\
& \widetilde{\mathcal{R}}_{A} \widetilde{+}\left[\left[\widetilde{\mathcal{R}}_{B}\right] \simeq\left[\widetilde{\mathcal{R}}_{D}\right]\right]=\left[\begin{array}{llll}
1 & 0 & 0 & 1 \\
0 & 1 & 1 & 0
\end{array}\right] \\
& {\left[\left[\widetilde{\mathcal{R}}_{A}\right] \widetilde{+}\left[\widetilde{\mathcal{R}}_{B}\right]\right]=\left[\begin{array}{llll}
1 & 1 & 1 & 1 \\
0 & 1 & 1 & 1
\end{array}\right],\left[\left[\widetilde{\mathcal{R}}_{A}\right] \widetilde{+}\left[\widetilde{\mathcal{R}}_{D}\right]\right]=\left[\begin{array}{llll}
1 & 0 & 0 & 1 \\
1 & 1 & 1 & 0
\end{array}\right] \text {, and }} \\
& {\left[\left[\widetilde{\mathcal{R}}_{A}\right] \widetilde{+}\left[\widetilde{\mathcal{R}}_{B}\right]\right] \simeq\left[\left[\widetilde{\mathcal{R}}_{A}\right] \widetilde{+}\left[\widetilde{\mathcal{R}}_{D}\right]\right]=\left[\begin{array}{llll}
1 & 0 & 0 & 1 \\
0 & 1 & 1 & 0
\end{array}\right]} \\
& {\left[\widetilde{\mathcal{R}}_{A}\right] \widetilde{+}\left(\left[\widetilde{\mathcal{R}}_{B}\right] \simeq\left[\widetilde{\mathcal{R}}_{D}\right]\right)=\left(\left[\widetilde{\mathcal{R}}_{A}\right] \widetilde{+}\left[\widetilde{\mathcal{R}}_{B}\right]\right) \simeq\left(\left[\widetilde{\mathcal{R}}_{A}\right] \widetilde{+}\left[\widetilde{\mathcal{R}}_{D}\right]\right) .}
\end{aligned}
$$

Now we define product of soft real point matrices as:

Definition 3.2. Let $\left[\widetilde{\mathcal{R}}_{A}\right],\left[\widetilde{\mathcal{R}}_{B}\right] \in M(\widetilde{\mathcal{R}})_{m \times n}$. Then

(i) The common product of $\left[\widetilde{\mathcal{R}}_{A}\right]$ and $\left[\widetilde{\mathcal{R}}_{B}\right]$ is defined by

$\sim:\left[\widetilde{\mathcal{R}}_{A}\right]_{m \times n} \sim\left[\widetilde{\mathcal{R}}_{B}\right]_{m \times n} \rightarrow\left[\widetilde{\mathcal{R}}_{C}\right]_{m \times n^{2}},\left[a_{i j}\right] \widetilde{r}\left[b_{i k}\right]=\left[c_{i p}\right]$.

Where $c_{i p}=\max \left\{a_{i j}, b_{i k}\right\}$ such that $p=n(j-1)+k$.

(ii) The restricted product of $\left[\widetilde{\mathcal{R}}_{A}\right]$ and $\left[\widetilde{\mathcal{R}}_{B}\right]$ is defined by

$\widetilde{\mathcal{R}}^{\mathcal{R}}:\left[\widetilde{\mathcal{R}}_{A}\right]_{m \times n}{\stackrel{{ }^{\mathcal{R}}}{ }}\left[\widetilde{\mathcal{R}}_{B}\right]_{m \times n} \rightarrow\left[\widetilde{\mathcal{R}}_{C}\right]_{m \times n^{2}}, \quad\left[a_{i j}\right] \widetilde{\sim}_{\mathcal{R}}\left[b_{i k}\right]=\left[c_{i p}\right]$

Where $c_{i p}=\min \left\{a_{i j}, b_{i k}\right\}$ such that $p=n(j-1)+k$.

Example 3.5. We consider the following soft real point matrices

$$
\begin{aligned}
& {\left[\widetilde{\mathcal{R}}_{B}\right]=\left[\begin{array}{llll}
1 & 0 & 0 & 1 \\
1 & 0 & 1 & 0
\end{array}\right] \text { and }\left[\widetilde{\mathcal{R}}_{A}\right]=\left[\begin{array}{llll}
1 & 0 & 1 & 0 \\
1 & 0 & 0 & 1
\end{array}\right] \text {. Then }} \\
& {\left[\widetilde{\mathcal{R}}_{A}\right] \sim\left[\widetilde{\mathcal{R}}_{B}\right]=\left[\widetilde{\mathcal{R}}_{C}\right]=\left[\begin{array}{llllllll}
c_{11} & c_{12} & c_{13} & c_{14} & \ldots & \ldots & c_{115} & c_{116} \\
c_{21} & c_{22} & c_{23} & c_{24} & \ldots & \ldots & c_{215} & c_{216}
\end{array}\right] \text {. }} \\
& \text { Where } c_{i p}=\max \left\{a_{i j}, b_{i k}\right\} \text { such that } p=n(j-1)+k .(\text { Here } n=4) \\
& \text { - If } i=1 \text { and } j=1 \text {, then } p=4(1-1)+k \text {, } \\
& \text { for } k=1 \text {, then } \max \left\{a_{11}, b_{11}\right\}=c_{1 p}, p=4(1-1)+1=1 \text {, } \\
& \text { so } \max \left\{a_{11}, b_{11}\right\}=c_{11} \text {, } \\
& \text { for } k=2 \text {, then } \max \left\{a_{11}, b_{12}\right\}=c_{1 p}, p=4(1-1)+2=2 \text {, } \\
& \text { so } \max \left\{a_{11}, b_{12}\right\}=c_{12} \text {, }
\end{aligned}
$$


for $k=3$, then $\max \left\{a_{11}, b_{13}\right\}=c_{1 p}, p=4(1-1)+3=3$, so $\max \left\{a_{11}, b_{13}\right\}=c_{13}$,

for $k=4$, then $\max \left\{a_{11}, b_{14}\right\}=c_{1 p}, p=4(1-1)+4=4$, so $\max \left\{a_{11}, b_{14}\right\}=c_{14}$,

- If $i=1$ and $j=2$, then $p=4(2-1)+k$,

for $k=1$, then $\max \left\{a_{12}, b_{11}\right\}=c_{1 p}, p=4(2-1)+1=5$, so $\max \left\{a_{12}, b_{11}\right\}=c_{15}$,

for $k=2$, then $\max \left\{a_{12}, b_{12}\right\}=c_{1 p}, p=4(2-1)+2=6$, so $\max \left\{a_{12}, b_{12}\right\}=c_{16}$,

for $k=3$, then $\max \left\{a_{12}, b_{13}\right\}=c_{1 p}, p=4(2-1)+3=7$, so $\max \left\{a_{12}, b_{13}\right\}=c_{17}$,

for $k=4$, then $\max \left\{a_{12}, b_{14}\right\}=c_{1 p}, p=4(2-1)+4=8$, so $\max \left\{a_{12}, b_{14}\right\}=c_{18}$,

- If $i=1$ and $j=3$, then $p=4(3-1)+k$,

for $k=1$, then $\max \left\{a_{13}, b_{11}\right\}=c_{1 p}, p=4(3-1)+1=9$,

so $\max \left\{a_{13}, b_{11}\right\}=c_{19}$,

for $k=2$, then $\max \left\{a_{13}, b_{12}\right\}=c_{1 p}, p=4(3-1)+2=10$,

so $\max \left\{a_{13}, b_{12}\right\}=c_{110}$,

for $k=3$, then $\max \left\{a_{13}, b_{13}\right\}=c_{1 p}, p=4(3-1)+3=11$

so $\max \left\{a_{13}, b_{13}\right\}=c_{111}$,

for $k=4$, then $\max \left\{a_{13}, b_{14}\right\}=c_{1 p}, p=4(3-1)+4=12$

so $\max \left\{a_{13}, b_{14}\right\}=c_{112}$,

- If $i=1$ and $j=4$, then $p=4(4-1)+k$,

for $k=1$, then $\max \left\{a_{14}, b_{11}\right\}=c_{1 p}, p=4(4-1)+1=13$,

so $\max \left\{a_{14}, b_{11}\right\}=c_{113}$,

for $k=2$, then $\max \left\{a_{14}, b_{12}\right\}=c_{1 p}, p=4(4-1)+2=14$,

so $\max \left\{a_{14}, b_{12}\right\}=c_{114}$,

for $k=3$, then $\max \left\{a_{14}, b_{13}\right\}=c_{1 p}, p=4(4-1)+3=15$,

so $\max \left\{a_{14}, b_{13}\right\}=c_{115}$,

for $k=4$, then $\max \left\{a_{14}, b_{14}\right\}=c_{1 p}, p=4(4-1)+4=16$,

so $\max \left\{a_{14}, b_{14}\right\}=c_{116}$.

In the same way if $i=2$

So, $\left[\widetilde{\mathcal{R}}_{A}\right] \sim\left[\widetilde{\mathcal{R}}_{B}\right]=\left[\begin{array}{llllllllllllllll}1 & 1 & 1 & 1 & 1 & 0 & 0 & 1 & 1 & 1 & 1 & 1 & 1 & 0 & 0 & 1 \\ 1 & 1 & 1 & 1 & 1 & 0 & 1 & 0 & 1 & 0 & 1 & 0 & 1 & 1 & 1 & 1\end{array}\right]$.

Similarly,

$\left[\widetilde{\mathcal{R}}_{A}\right] \widetilde{\sim}_{\mathcal{R}}\left[\widetilde{\mathcal{R}}_{B}\right]=\left[\begin{array}{llllllllllllllll}1 & 0 & 0 & 1 & 0 & 0 & 0 & 0 & 1 & 0 & 0 & 1 & 0 & 0 & 0 & 0 \\ 1 & 0 & 1 & 0 & 0 & 0 & 0 & 0 & 0 & 0 & 0 & 0 & 1 & 0 & 1 & 0\end{array}\right]$.

Proposition 3.10. Let $\left[\widetilde{\mathcal{R}}_{A}\right],\left[\widetilde{\mathcal{R}}_{B}\right] \in M(\widetilde{\mathcal{R}})_{m \times n}$. Then

(i) $\left[\left[\widetilde{\mathcal{R}}_{A}\right] \sim\left[\widetilde{\mathcal{R}}_{B}\right]\right]^{c}=\left[\widetilde{\mathcal{R}}_{A}\right]^{c} \sim_{\mathcal{R}}\left[\widetilde{\mathcal{R}}_{B}\right]^{c}$

(ii) $\left[\left[\widetilde{\mathcal{R}}_{A}\right] \widetilde{\mathcal{R}}_{\mathcal{R}}\left[\widetilde{\mathcal{R}}_{B}\right]\right]^{c}=\left[\widetilde{\mathcal{R}}_{B}\right]^{c} \sim\left[\widetilde{\mathcal{R}}_{A}\right]^{c}$.

Proof. (i) For all $i$ and $j$,

$\left[\left[\widetilde{\mathcal{R}}_{A}\right] \sim\left[\widetilde{\mathcal{R}}_{B}\right]\right]^{c}=\left[\widetilde{\mathcal{R}}_{C}\right]^{c}=\left[c_{i p}\right]^{c}$,

$\left[c_{i p}\right]^{c}=\left[\max \left\{a_{i j}, b_{i k}\right\}\right]^{c}$, such that $p=n(j-1)+k$

$=\left[1-\max \left\{a_{i j}, b_{i k}\right\}\right]$

$=\left[\min \left\{1-a_{i j}, 1-b_{i k}\right\}\right]=\left[\widetilde{\mathcal{R}}_{A}\right]^{c} \sim_{\mathcal{R}}\left[\widetilde{\mathcal{R}}_{B}\right]^{c}$

(ii) Similarly to $(i)$. 


\section{References}

[1] Aygunoglu A., Aygun H., Some notes on soft topological spaces, Neural Computing and Applications, DOI:10. 1007/s00521-011-0722-3, 2011.

[2] Cagman N., Karatas S., Enginoglu S., Soft topology, Computers and Mathematics with Applications, (2011), $351-358$

[3] Hussain S., Ahmad B., Some properties of soft topological spaces, Computers and Mathematics with Applications, 62(2011), 4058-4067.

[4] Hussain S., Ahmad B., Soft separation axioms in soft topological spaces, Hacettepe Journal of Mathematics and Statistics, 44(3)(2015), 559-568.

[5] Hussain S., A note on soft connectedness, Journal of Egyptian Mathematical Society, 23(1)(2015), 6-11.

[6] Hussain S., Akiz, H. F., Alajlan A. I., On algebraic properties of soft real points,(Submitted)

[7] Maji P. K., Roy A. R., Biswas R., An application of soft sets in a decision making problem, Computers and Mathematics with Applications, 44(2002), 1077-1083.

[8] Molodtsov D., Soft set theory-first results, Computers and Mathematics with Applications, 37(1999), 19 -31.

[9] Tanay B., Kandemir M. B,. Topological structure of fuzzy soft sets, Computers and Mathematics with Applications, 61(10)(2011), 2952-2957.

[10] Zadeh L.A., Fuzzy sets, Inform. Control. 8 (1965) 338-353.

[11] Zorlutuna I., Akdag M., Min W. K., Atmaca S., Remarks on soft topological spaces, Annals of Fuzzy Mathematics and Informatics, Vol.3 No.2 (2012) 171-185. 